\title{
An Approach to Modal Analysis of Power System Angle Stability
}

\author{
A. Roth, D. Ruiz-Vega, Student Member, IEEE, D. Ernst, Student Member, IEEE, C. Bulac,
}

M. Pavella, Fellow, IEEE, and G. Andersson, Fellow, IEEE

\begin{abstract}
An approach to modal analysis and modal identification is proposed, capable of complementing the panoply of existing methods. It is based on a hybrid time-domain - direct transient stability method called SIME (for SIngle Machine Equivalent). In short, SIME uses a conventional transient stability program to transform the time varying parameters of the system into those of a one-machine infinite bus (OMIB) equivalent system. The representations of this OMIB allow substantial reduction of the original problem's dimensionality. Many important advantages may result. For example, the multimachine system unstable equilibrium point (UEP) can readily be derived from the OMIB UEP, which is calculated analytically and unambiguously in a two-dimensional space. Further, the interplay between multimachine and OMIB characteristics and their complementary properties provides a better understanding and handling of damping, inter-area oscillations and their control. More generally, modal analysis and modal identification tasks get closer to each other and easier to handle. The paper essentially focuses on the approach as such rather than on potential applications. Simulations carried out on a 3-machine system illustrate main features.
\end{abstract}

Index Terms-modal analysis; model identification; transient stability control; preventive control; damping; oscillations.

\section{INTRODUCTION}

Power system angle stability covers two aspects: small-signal (small-disturbance) stability and transient (large-disturbance) stability. An important issue common to both aspects is power system oscillations. Their study encompasses modal analysis and modal identification [1].

Modal analysis of angle stability deals with the determination of the characteristic modes of a system model linearized about its stable or unstable equilibrium points (SEP or UEP). It mainly consists of computing eigenvalues, eigenvectors, participation factors, and the like. In particular, analysis of these quantities allows uncovering the participation (natural modes of oscillation) of the various machines losing synchronism, as for example in an

A. Roth is with the Dept of Electric Power Systems, Royal Institute of Technology, Stockholm, Sweden; on leave from the University of Liège.

D. Ernst, D. Ruiz-Vega and M. Pavella are with the Dept of Electrical Engg, University of Liège, Sart-Tilman B28, B-4000 Liège Belgium (email: mania.pavella@ulg.ac.be).

D. Ernst is a Research Fellow, FNRS

C. Bulac is with the Polytechnic University of Bucharest. Dept of Electric Power Engg; on leave from the University of Liège.

G. Andersson is with the Power Systems Group, ETH, CH-8092 Zurich, Switzerland. inter-area mode oscillation [2]. While post-disturbance SEPs are generally easy to determine, computation of UEPs has long been considered as a problematic task [3,4]. Modal identification, on the other hand, involves the determination of characteristic modes from large-disturbance dynamic behavior obtained via transient stability simulations.

The approach proposed in this paper relies on the hybrid transient stability method called SIME (for SIngle Machine Equivalent). In short, SIME uses a numerical program to get the system time varying parameters and to transform them into those of a one-machine infinite bus (OMIB) equivalent system, suitably defined. By refreshing the OMIB parameters at each time-step of the program along the post-disturbance trajectory, SIME provides an accurate picture of the multimachine system trajectory. Besides, the equal-area criterion determines analytically necessary and sufficient borderline (in)stability conditions in a twodimensional space, and corresponding conditions in the multimachine space. The coordinates of "SIME's UEP" are by construction close to the multimachine system UEP. Hence, using them as starting conditions makes the minimization procedure search significantly easier and faster [5].

Further, comparing modal analysis performed about SIME's and system's UEPs provides interesting results. Stabilization procedures relying on participation factors and on SIME are also worth comparing.

The proposed approach is general and free from any restrictions regarding power system modeling and size, contingency scenario or type of instability (first- or multi-swing, etc). It can therefore yield easy sensitivity analyses, for example of the influence of various parameters (e.g. damping) on system stability.

The modal analysis and modal identification approaches proposed in this paper are different from the existing conventional ones. It is expected that they will provide additional insight into an old but timely problem and new means of tackling it.

\section{FUNDAMENTALS OF SIME}

\subsection{Notation}

All symbols are fully defined at the place where they are first introduced. Some of the more frequently used ones are collected below.

CCT Critical Clearing Time

CM Critical Machine

CT Clearing Time

NM Non-critical Machine 
OMIB One-Machine Infinite Bus

$P_{e}, P_{m}$ respectively OMIB electrical output power and mechanical input power

$P_{a} \quad$ OMIB accelerating power: $P_{a}=P_{m}-P_{e}$

SEP Stable Equilibrium Point

SIME SIngle Machine Equivalent

UEP Unstable Equilibrium Point

$\delta_{r} \quad$ OMIB "return" angle, i.e., OMIB maximum angular deviation on a stable trajectory

$\delta_{u} \quad$ OMIB unstable angle

$t_{r} \quad$ "return" time, i.e. time to (reach) stability conditions, i.e. to reach $\delta_{r}$

$t_{u} \quad$ time to (reach) instability conditions, i.e. to reach $\delta_{u}$

\subsection{Foundations}

The multimachine power system parameters provided by a time-domain program are transformed into those of a onemachine infinite bus (OMIB) system, and refreshed at each time step of the program. Further, at each time step, the stability of the OMIB is explored by the Equal Area Criterion (EAC); the procedure is stopped as soon as the (in)stability conditions of the EAC are reached (see below).

More precisely, after a contingency inception and its clearance, SIME drives a time-domain program so as to accomplish the following tasks: identify the critical and non critical machines and aggregate them into two groups; replace these groups by successively a two-machine, then an OMIB equivalent system; assess transient stability of this OMIB, using the EAC [6]. The various steps of the method are briefly described below and illustrated in Figs 1 drawn for the 3-machine system simulated in Section 5. For more details about SIME, see [7].

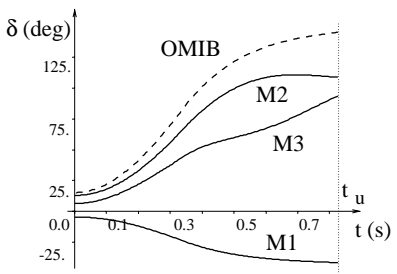

(a) OMIB and 3-machine systems swing curves (b) OMIB $P-\delta$ curve

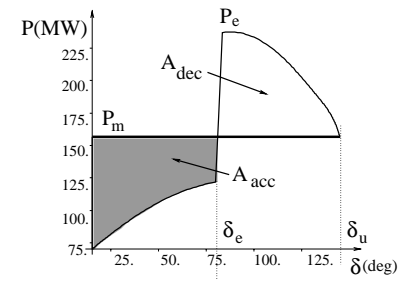

Fig. 1. Swing curves and OMIB $P-\delta$ representation of the 3 -machine system considered in Section 5. Contingency \# 2; $\mathrm{CCT}=334 \mathrm{~ms} ; \mathrm{CT}=335 \mathrm{~ms}$

\subsection{Identification of the critical machines (CMs)}

By definition, the critical machines are those which cause the system loss of synchronism. To identify them, SIME drives the time-domain (T-D) transient stability program, and, as soon as the system enters the post-fault phase, it starts considering a few candidate decomposition patterns, by: (i) sorting the machines according to their rotor angles; (ii) identifying the very first largest rotor angular deviations ("distances") between adjacent machines; (iii) considering as candidate CMs those which are above each one of the (say, 5) largest distances. The procedure is carried out until a candidate group of CMs and corresponding OMIB reaches the unstable conditions (3) defined below. It is then declared to be the critical OMIB of concern or simply the $\mathrm{OMIB}^{1}$.

\subsection{OMIB parameters, margins and by-products}

The OMIB parameters $\delta, \omega, M, P_{m}, P_{e}$ are computed from the corresponding individual machines parameters, using the concept of partial center of angle. On the other hand, the EAC states that the stabiltiy margin is the excess of the decelerating area over the accelerating area (Fig. 1b). Accordingly, the following analytical expressions for unstable and stable margins are derived:

$$
\begin{aligned}
\eta_{u} & =-\frac{1}{2} M \omega_{u}^{2} \\
\eta_{s t} & =\int_{\delta_{r}}^{\delta_{u}}\left|P_{a}\right| d \delta .
\end{aligned}
$$

In these expressions,

- $P_{a}$ is the accelerating power: $P_{a}=P_{m}-P_{e}$,

- subscript $u$ (for unstable) refers to the angle $\delta_{u}$, speed $\omega_{u}$, and time $t_{u}$ when the OMIB instability conditions are met:

$$
P_{a}\left(t_{u}\right)=0 ; \dot{P}_{a}\left(t_{u}\right)>0
$$

- subscript $r$ (for return) refers to the "return" angle $\delta_{r}$ and time $t_{r}$ where $\delta$ starts decreasing and $\omega$ vanishes (OMIB stability conditions):

$$
\omega\left(t_{r}\right)=0 ; P_{a}\left(t_{r}\right)<0 .
$$

Figs 1 illustrate SIME in an unstable case.

\subsection{Stabilization}

According to SIME, stabilizing an unstable contingency scenario consists of cancelling out its (negative) margin. According to EAC, in terms of the OMIB parameters, this corresponds to decreasing the OMIB mechanical power, see Fig. $1 \mathrm{~b}$ (unless the instability is of the back-swing type, for which the OMIB mechanical power should be increased). The application of the EAC can guide effectively the search of the amount, $\Delta P_{m}$, needed to cancel out the margin.

In terms of the multimachine system parameters, it can be shown that, in turn,

$$
\Delta P_{m}=\Delta P_{C}=-\Delta P_{N}
$$

where $P_{C}$ (respectively $P_{N}$ ) stands for generation power of the CMs (respectively of the NMs) [7].

In other words, SIME determines how much generation to shift from CMs to NMs. Further, to determine how to report the total

\footnotetext{
${ }^{1}$ Note that the above criterion for identifying the CMs and corresponding OMIB obeys the necessary and sufficient conditions derived from EAC and expressed by (3). This unambiguous identification of the CMs is a major advantage of hybrid one-machine equivalent methods over hybrid multimachine methods. Besides, the criterion is free from any pragmatic consideration, unlike T-D methods which call upon pragmatic criteria to detect instability. Finally, the procedure is computationally very little demanding: it involves very unexpensive computations and, in addition, it allows saving number and duration of T-D simulations.
} 
generation decrease, $\Delta P_{C}$, on the various CMs, SIME may invoke the "degree of criticality" of each CM. For the $i$-th CM, this latter is considered to be proportional to its inertia, $H_{i}$, and to its "electrical distance", $d_{i}$, measuring its angular deviation with respect to a reference (for example, with respect to the most advanced NM), assessed at $t_{u}$. It thus comes:

$$
\frac{\Delta P_{C i}}{\Delta P_{C k}}=\frac{H_{i} d_{i}}{H_{k} d_{k}} ; \Delta P_{C}=\sum_{i \in=C} \Delta P_{C i}
$$

\section{CONVENTIONAL SMALL-SIGNAL ANALYSIS}

\subsection{Fundamentals of modal analysis $[1,8,9]$}

The non-linear model describing the dynamics of a power system comprises a set of differential and algebraic equations:

$$
\begin{aligned}
\dot{\boldsymbol{x}} & =\boldsymbol{f}(\boldsymbol{x}, \boldsymbol{y}) \\
\mathbf{0} & =\boldsymbol{g}(\boldsymbol{x}, \boldsymbol{y})
\end{aligned}
$$

where $\boldsymbol{x}$ and $\boldsymbol{y}$ are the vectors of state variables and algebraic variables, respectively.

A conventional modal analysis is performed by linearizing the system equations (7) about an equilibrium point, $\boldsymbol{x}_{o}$, defined by:

$$
\dot{\boldsymbol{x}}_{o}=\boldsymbol{f}\left(\boldsymbol{x}_{o}\right)=\mathbf{0} .
$$

Small disturbances around $\boldsymbol{x}_{o}$ can then be described by

$$
\Delta \dot{\boldsymbol{x}}=\boldsymbol{A} \Delta \boldsymbol{x}
$$

where $\boldsymbol{A}$ is the system state matrix defined by

$$
\boldsymbol{A}=[A i j]=\left[\left.\frac{\partial f_{i}}{\partial x_{j}}\right|_{\boldsymbol{x}=\boldsymbol{x}_{\circ}}\right]
$$

\subsection{Modal analysis performance measures}

The characteristic modes of eq. (10) have the general form

$$
\varphi_{i} e^{\lambda_{i} t}
$$

where $\varphi_{i}$ is the characteristic vector (or right eigenvector) and $\lambda_{i}$ is the corresponding eigenvalue. Eigenvalues are solutions of the characteristic equation

$$
\operatorname{det}(\boldsymbol{A}-\lambda \boldsymbol{I})=0,
$$

and the right eigenvectors are column vectors satisfying

$$
\boldsymbol{A} \varphi_{i}=\lambda_{i} \varphi_{i} .
$$

The right eigenvector associated with each mode defines the relative distribution of the mode throughout the system dynamic states.

On the other hand, the left eigenvectors defined by

$$
\psi_{i} \boldsymbol{A}=\lambda_{i} \psi_{i}
$$

provide, together with the right eigenvectors, the participation factors. In particular, the participation factor $p_{i j}$ expressed by

$$
p_{i j}=\frac{\partial \lambda_{i}}{\partial a_{j j}}
$$

measures the sensitivity of $\lambda_{i}$ to the change in the $j$-th diagonal element $a_{j j}$. It also measures the relative participation of the $j$ th state variable in the $i$-th mode and vice versa. In other words, it gives an indication of the sensitivity of a system physical component to a mode. A direct practical application is the consideration of participation factors in the stabilization procedure described in $\S 2.5$ : instead of distributing power on critical machines proportionally to their "degree of criticality", $H_{i} * d_{i}$ (see eq. (6)), one may think of distributing it proportionally to the corresponding participation factors, $P F_{i}{ }^{2}$ :

$$
\frac{\Delta P_{C i}}{\Delta P_{C k}}=\frac{P F_{i}}{P F_{k}} ; \Delta P_{C}=\sum_{i \in C} \Delta P_{C i} .
$$

\section{PROPOSED APPROACH}

\subsection{Search of equilibrium points}

For small-signal analysis, calculating system's equilibrium points is a rather easy task, but for large-disturbance analysis the search of the post-fault unstable equilibrium point (UEP) may be problematic. Indeed, numerical instabilities linked to the physical unstable nature of UEP make its search difficult through conventional minimization procedures. These procedures may even converge to the SEP instead of the UEP sought, unless the starting point is close enough to this UEP [10].

The transformation of the multimachine angle state space into the OMIB uni-dimensional one and the interplay between them renders the UEP search straightforward and unambiguous. The reasoning underlying this search is as follows. For a given contingency and its clearing time, CT, the instability conditions (3) define the unstable angle, $\delta_{u}$, and corresponding time $t_{u}$. Now, by definition, when the clearing time gets close to the critical clearing time, the unstable angle gets close to its maximum value and almost coincides with the maximum stable angle, $\delta_{r}$, obeying the stability conditions (4). In other words, for CT $=$ CCT : $\delta_{u}=\mathrm{UEP}_{O M I B} ; \omega_{O M I B}=0$.

The search of the multimachine UEP derives from the above considerations. It is summarized in the following four steps [5]:

(i) run SIME to find the contingency CCT

(ii) using a slightly larger clearing time $(\mathrm{CT}=\mathrm{CCT}+\epsilon$, where $\epsilon$ is a small quantity), run SIME to determine the corresponding time to instability, $t_{u}$

(iii) at $t_{u}$, identify the corresponding angles of the multimachine system, $\delta_{0 u i}(i=1,2, \ldots, n)$

(iv) using as initial values these angles $\delta_{0 u i}$, search the components $\delta_{u i}$ of the multimachine UEP via a standard minimization procedure.

Ref. [5] has used a simple iterative perturbation procedure in the case of the 3-machine system. In what follows, we will improperly denote the angles $\delta_{0 u i}$ as being the components of the "SIME UEP", whereas the angles $\delta_{u i}$ are the components of the multimachine system UEP.

${ }^{2}$ Actually, the authors of [4] proceed in an opposite way: they propose to identify the system CMs based on the size of their corresponding participation factors. However, it is not always easy to determine the border between CMs and NMs. 
The above general procedure applies to the search of both prefault and post-fault SEPs. Note that in the particular case of simplified power system modeling SEPs may simply be computed by a conventional load flow program.

\subsection{Modal analysis performance measures}

Using the definitions of $\oint 3.2$ and linearizing the multimachine power system about its SEPs and UEPs, computed according to $\S 4.1$, provides readily the corresponding system eigenvalues. Their comparison with the corresponding SIME's eigenvalues computed in a similar way provides interesting results.

Similarly, the eigenvectors and corresponding participation factors are computed. Further, the relative size of the participation factors corresponding to the system machines are again compared to the "degree of criticality" $H_{i} * d_{i}$ as provided by SIME.

\subsection{Note on SIME-based modal identification}

Modal identification involves the determination of system dynamic behavior, possibly from measurements, or transient stability simulations using non-linear models. Among many signal analysis tools used to this end, Prony analysis is acknowledged to be a powerful and useful technique [1]. For example, in [11], applying a multichannel Prony analysis to the swing curves of 4 selected generators, out of 798 available in the system, gives a good assessment of the damping. However, the problem of selecting the correct generators remains an open question. Using SIME, the dynamics of the whole system is represented by the single OMIB swing curve; this solves the problem of selecting the correct generators and makes the application of a single channel Prony analysis much easier. Even in a system as simple as the 3 -machine one, Figs 2 suggest how easily the system damping can be visualized and analyzed.

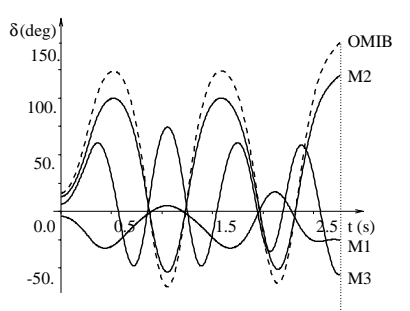

(a) Machines and OMIB swing curves: $\mathrm{CT}=292 \mathrm{~ms}$ (unstable case)

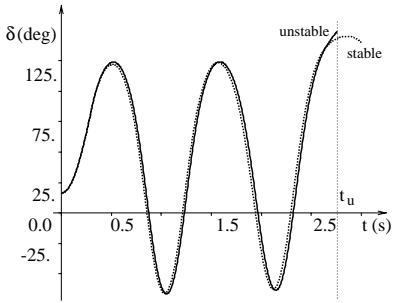

(b) OMIB swing curves; $\mathrm{CT}=292 \mathrm{~ms}$ (unstable case) and CT=294 $\mathrm{ms}$ (borderline stable case)
Fig. 2. Swing curves for contingency \# 5;

\section{ILLUSTRATIONS}

\subsection{System description}

All simulation results reported in this section are taken from [5]. They are obtained with the 3-machine, 9-bus system sketched in Fig. 3 . The time-domain program used here by SIME as well as for computing eigenproperties of the 3-machine system is written in MATLAB [12].

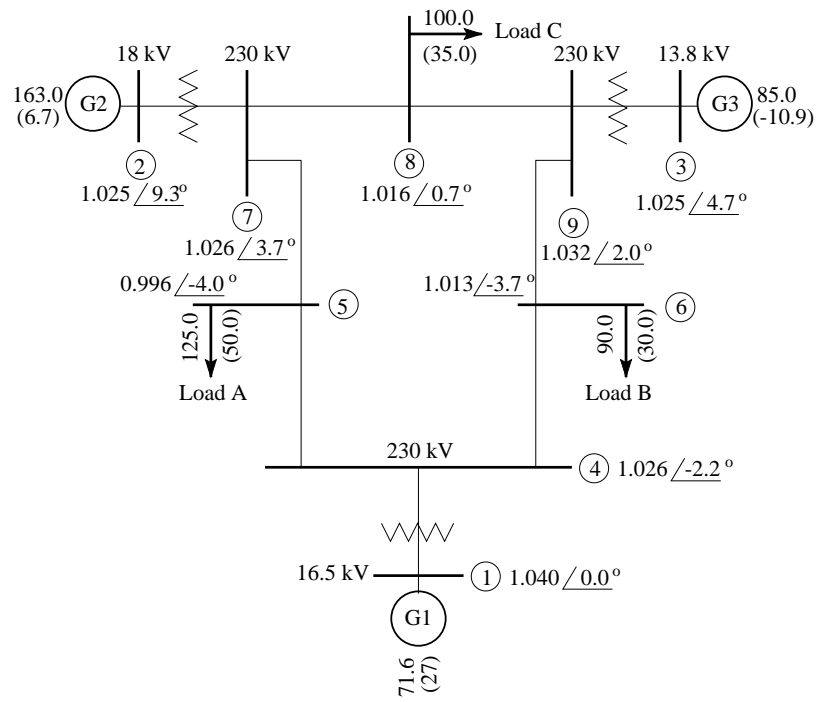

Fig. 3. One-line diagram of the 3-machine, 9-bus test system. $H_{1}=23.64 \mathrm{~s}, H_{2}=6.40 \mathrm{~s}, H_{3}=3.01 \mathrm{~s}$

\subsection{Post-fault SEP and eigenproperties}

In the particular case of simplified power system modeling, the SEP is readily computed either by following the procedure of $\S 4.1$ or simply by running a load flow program.

As an example, the SEP obtained with both approaches for the post-fault configuration resulting by tripping line 5-7 is given by

$$
\begin{aligned}
\mathbf{x}_{0} & =\left[\begin{array}{llllll}
\omega_{10} & \delta_{10} & \omega_{20} & \delta_{20} & \omega_{30} & \delta_{30}
\end{array}\right]^{T} \\
& =\left[\begin{array}{llllll}
0.0 & 0.0 & 0.0 & 41.73 & 0.0 & 26.55
\end{array}\right]^{T}
\end{aligned}
$$

where angular speeds are expressed in $\mathrm{rad} / \mathrm{s}$ and angles in degrees. Linearizing about the above SEP (cfr eq. (10)), one gets the corresponding eigenvalues

$$
\lambda_{\mathrm{SEP}}=\left[\begin{array}{llllll}
0 & 0 & j 6.09 & -j 6.09 & j 12.90 & -j 12.90
\end{array}\right]^{T} .
$$

Note that there are 6 eigenvalues, since there are 6 states. But, actually, two eigenvalues are zero, since the simplified system modeling has $2(n-1)=4$ independent state variables. Note also that the other four eigenvalues occur in conjugated pairs with zero real part, since damping is neglected.

\subsection{Contingency scenarios: description, UEPs and corresponding eigenproperties}

The contingencies considered consist of three-phase short circuits $(3 \phi \mathrm{SCs})$ applied at each one of the buses $4,5,6,7,8,9$, and cleared by opening one line connected to this bus (see Fig.3). This creates 12 contingencies.

According to $\S 4.1$, the search of the corresponding SIME's UEP (computed at $t_{u}$ and denoted $\delta_{0 u}$ ) and system's UEP requires computation of the corresponding CCTs, and use of a clearing time $\mathrm{CT}=\mathrm{CCT}+\epsilon$. This yields an unstable simulation and corrsponding system's CMs; the unstable margin is also 
Table 1. Simulation results of unstable contingency scenarios

\begin{tabular}{|c|c|c|c|c|c|c|c|c|c|c|c|c|}
\hline 1 & 2 & 3 & 4 & 5 & 6 & 7 & 8 & 9 & 10 & 11 & 12 & 13 \\
\hline \multicolumn{3}{|c|}{ Contingency } & \multicolumn{7}{|c|}{ SIME } & \multicolumn{3}{|c|}{ 3-machine system } \\
\hline \# & $\begin{array}{c}\text { Applied } \\
\text { at } \\
\text { bus \# }\end{array}$ & $\begin{array}{c}\text { Cleared } \\
\text { by } \\
\text { op.line \# }\end{array}$ & $\begin{array}{l}\text { CCT } \\
\text { (ms) }\end{array}$ & $\begin{array}{c}\mathrm{CT}= \\
\mathrm{CCT}+\epsilon\end{array}$ & $\begin{array}{c}\eta \\
(\mathrm{rad} / \mathrm{s})^{2}\end{array}$ & $\mathrm{CM}(\mathrm{s})$ & $\begin{array}{l}\text { UEP } \\
\left({ }^{\circ}\right)\end{array}$ & $\lambda$ & $\mathrm{PF}$ & $\begin{array}{l}\text { UEP } \\
\left({ }^{\circ}\right)\end{array}$ & $\lambda$ & $\mathrm{PF}$ \\
\hline 1 & 7 & $5-7$ & 171 & 172 & -0.04 & $\mathrm{M}_{2}, \mathrm{M}_{3}$ & $\begin{aligned}-39.1 & (0) \\
103.7 & (142.8) \\
86.8 & (125.2)\end{aligned}$ & $\begin{array}{c}6.38 \\
0 \\
j 8.66\end{array}$ & $\begin{array}{l}0.49 \\
1.00 \\
0.81\end{array}$ & $\begin{aligned}-42.6 & (0) \\
109.1 & (151.7) \\
85.2 & (127.8)\end{aligned}$ & $\begin{array}{c}6.56 \\
0 \\
j 8.31\end{array}$ & $\begin{array}{l}0.52 \\
1.00 \\
0.81\end{array}$ \\
\hline 2 & 5 & $5-7$ & 334 & 335 & -0.17 & $\mathrm{M}_{2}, \mathrm{M}_{3}$ & $\begin{aligned}-41.3 & (0) \\
108.6 & (149.9) \\
93.5 & (134.8)\end{aligned}$ & $\begin{array}{c}6.83 \\
0 \\
j 8.46\end{array}$ & $\begin{array}{l}0.57 \\
1.00 \\
0.94\end{array}$ & \begin{tabular}{|rl}
-32.6 & $(0)$ \\
119.1 & $(151.7)$ \\
95.2 & $(127.8)$
\end{tabular} & $\begin{array}{c}6.56 \\
0 \\
j 8.31\end{array}$ & $\begin{array}{l}0.52 \\
1.00 \\
0.81\end{array}$ \\
\hline 3 & 7 & $7-8$ & 185 & 186 & -3.26 & $\mathrm{M}_{2}$ & $\begin{array}{ll}-24.8 & (0) \\
112.7 & (137.5) \\
-44.7 & (-19.9)\end{array}$ & $\begin{array}{c}7.78 \\
0 \\
j 8.65\end{array}$ & $\begin{array}{l}0.15 \\
1.00 \\
0.04 \\
\end{array}$ & $\begin{aligned}-45.4 & (0) \\
96.7 & (142.0) \\
-37.5 & (7.9)\end{aligned}$ & $\begin{array}{c}7.79 \\
0 \\
j 8.79\end{array}$ & $\begin{array}{l}0.15 \\
1.00 \\
0.03\end{array}$ \\
\hline 4 & 8 & $7-8$ & 264 & 265 & -0.66 & $\mathrm{M}_{2}$ & $\begin{array}{ll}-20.5 & (0) \\
111.3 & (131.8) \\
-75.7 & (-55.2)\end{array}$ & $\begin{array}{c}7.53 \\
0 \\
j 6.72\end{array}$ & $\begin{array}{l}0.14 \\
1.00 \\
0.05\end{array}$ & $\begin{array}{ll}-25.7 & (0) \\
116.3 & (142.0) \\
-17.8 & (7.9)\end{array}$ & \begin{tabular}{|c|}
7.79 \\
0 \\
$j \quad 8.79$
\end{tabular} & $\begin{array}{l}0.16 \\
1.00 \\
0.03\end{array}$ \\
\hline 5 & 8 & 8-9 & 292 & 294 & -1.46 & $\mathrm{M}_{2}$ & $\begin{array}{ll}-25.5 & (0) \\
120.2 & (145.7) \\
-55.6 & (-30.1)\end{array}$ & $\begin{array}{c}8.06 \\
0 \\
j 8.30\end{array}$ & $\begin{array}{l}0.15 \\
1.00 \\
0.04\end{array}$ & \begin{tabular}{|rl}
-61.7 & $(0)$ \\
93.3 & $(155.0)$ \\
-36.8 & $(24.9)$
\end{tabular} & $\begin{array}{c}8.03 \\
0 \\
j 8.63\end{array}$ & $\begin{array}{l}0.16 \\
1.00 \\
0.03\end{array}$ \\
\hline 6 & 9 & $8-9$ & 239 & 240 & -3.52 & $\mathrm{M}_{3}$ & \begin{tabular}{|rl}
-27.3 & $(0)$ \\
33.8 & $(61.1)$ \\
142.5 & $(169.8)$
\end{tabular} & $\begin{array}{c}10.38 \\
0 \\
j 4.29\end{array}$ & $\begin{array}{l}0.09 \\
0.00 \\
1.00\end{array}$ & \begin{tabular}{|rl}
4.1 & $(0)$ \\
29.6 & $(25.5)$ \\
166.6 & $(162.5)$
\end{tabular} & $\begin{array}{c}10.69 \\
0 \\
j 6.48\end{array}$ & $\begin{array}{l}0.08 \\
0.01 \\
1.00\end{array}$ \\
\hline
\end{tabular}

computed, to allow assessing how close the corresponding machine angles are to the borderline conditions where $t_{r}$ and $t_{u}$ coincide, and so do $\delta_{u}$ and $\delta_{r}$. Further, the eigenvalues corresponding to SIME's and system's UEPs are computed. Finally, the participation factors relative to the angle with positive eigenvalue are also computed.

Because of space limitations, Table 1 gathers simulation results obtained with 6 out of the 12 contingencies, randomly selected. The other 6 contingencies exhibit similar behavior. The contents of the various columns are self explanatory, except for the following ones.

Column \#8: the angles are listed in absolute values and, between brackets, in values relative to the angle of the first, most advanced machine: this eases comparisons with values of column \#11, obtained by the minimization procedure initialized with the angles of column \#8.

Columns \#9 and 11: In the absence of damping, the eigenvalues are pairwise purely real $( \pm \alpha)$, zero (see comment following eq. (18)), or purely imaginary $( \pm j \beta)$; the three eigenvalues listed in columns 9 and 11 correspond to: $\alpha, 0, j \beta$.

Columns \#10 and 13: participation factors, computed via the SIME's and the system's UEPs respectively.

Inspection of the various columns conveys interesting information. In particular: the eigenvalues resulting from SIME's and system's UEPs are close to each other, and so are the participation factors.

The above observations are quite interesting, though not surprising since, by construction, SIME gives a faithful account of the multimachine dynamic behaviour. The advantage of using SIME's rather than system's UEPs to get system's eigenproperties is quite obvious; it allows one to get rid of the minimization procedure.

Figs 1, 2 and 4 illustrate interesting aspects of the simulation
Table 2. Power limits computed according to 4 patterns

\begin{tabular}{|c|c|c|c|c|}
\hline $\begin{array}{c}\text { Compensation } \\
\text { method }\end{array}$ & SIME & Part. fact. & Fifty-fifty & $\mathrm{CM}^{\mathrm{M}} \mathrm{M}_{2}$ \\
\hline $\mathrm{Ctg} \# 1: \mathrm{CCT}=171 \mathrm{~ms} ; \mathrm{CT}=240 \mathrm{~ms} ; \mathrm{CMs}: \mathrm{M}_{2}, \mathrm{M}_{3}$ \\
\hline$P_{\text {limit }}$ (MW) & 218.4 & 214.3 & 211.5 & $\mathbf{2 2 3 . 9}$ \\
\hline $\mathrm{Ctg} \# 2: \mathrm{CCT}=334 \mathrm{~ms} ; \mathrm{CT}=380 \mathrm{~ms} ; \mathrm{CMs}: \mathrm{M}_{2}, \mathrm{M}_{3}$ \\
\hline$P_{\text {limit }}(\mathrm{MW})$ & $\mathbf{2 3 9 . 6}$ & 239.1 & 238.9 & 238.4 \\
\hline
\end{tabular}

results. Figs 1 are relative to simulations with contingency \#2, while Fig. 2 and 4 are relative to contingency \# 5. Observe in Fig. 2a how clearer the multiswing phenomena are described by the OMIB swing curve, even in such a simple case. Incidentally, Fig. $2 \mathrm{~b}$ shows that the "time to instability", $t_{u}$, and the "return time", $t_{r}$, are very close; also, $\delta_{u}$ is very close to but slightly larger than $\delta_{r}$. Finally, Fig. 4 displays the participation factors computed for the system UEP and the SIME UEP (labelled "SIME" in the figure); obviously, they are very close to each other for the $\mathrm{CM}\left(\mathrm{M}_{2}\right)$ as well as for the $\mathrm{NMs}\left(\mathrm{M}_{3}, \mathrm{M}_{1}\right)$.

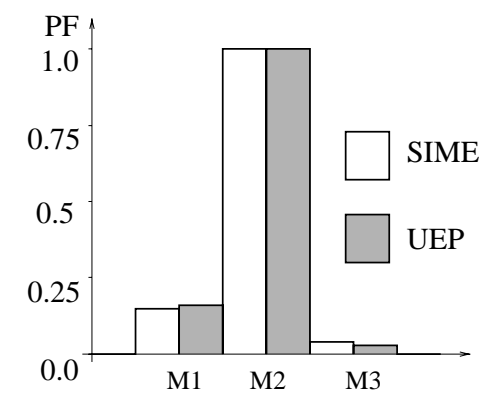

Fig. 4. Participation factors corresponding to the angle with the positive eigenvalue, computed for the system and SIME UEPs 


\subsection{Stabilization procedure}

The general procedure outlined in $\S 2.5$ may yield many variants, depending upon the way of reporting the total generation shifting on the various CMs, whenever there are many. For example, the total generation shifting may be distributed: (i) proportionally to the CMs' degree of criticality, see eq. (6); (ii) proportionally to the CMs' participation factors, see eq. (6'); (iii) equally on the various CMs; (iv) only on one CM (for example, the most advanced one).

The simulation results using the above four ways are summarized in Table 2 corresponding to two contingency scenarios having two CMs $\left(\mathrm{M}_{2}\right.$ and $\left.\mathrm{M}_{3}\right)$. The "quality" of these results depends upon the generation power limit of the system CMs (here, $\mathrm{M}_{2}$ and $\mathrm{M}_{3}$ ): the larger the $P_{\text {limit }}$ and the better. Note that, here, CT has been chosen quite larger than CCT in order to stress the system. According to this table, the SIME procedure described by eq. (6) shows to provide slightly better results than the participation factors, for ctg. \#2, whereas for ctg. \#1 shifting the generation of the most CM only provides a larger $P_{\text {limit }}$. Actually, the small size of the system, the small number of CMs and the simplified system modeling do not allow sound comparisons and conclusions; the reported results are merely for illustration.

\subsection{Influence of damping}

Among the various types of damping, the one used in this paragraph is the mechanical damping modeled by adding in the $i$-th dynamic equation a term $D \omega_{i}, D=D_{1}=D_{2}=D_{3}$. This simplified damping allows easy comparisons with the simulations reported so far, since it doesn't change the system SEPs and UEPs.

Eignvectors have thus been computed for various values of D. Such a typical example is given below. It concerns the eigenvectors obtained for a $3 \phi \mathrm{SC}$ applied at bus 6 and cleared by opening line 6-9 [5].

$$
\begin{aligned}
& \underset{\left(D_{i}=0\right)}{\lambda_{\text {(UEP) }}}=\left[\begin{array}{r}
6.95 \\
-6.95 \\
0 \\
0 \\
j 9.18 \\
-j 9.18
\end{array}\right] ; \quad \begin{array}{l}
\lambda_{\mathrm{UEP}(\mathrm{SIME})} \\
\left(D_{i}=0\right)
\end{array}=\left[\begin{array}{r}
7 \\
-7 \\
0 \\
0 \\
j 8.86 \\
-j 8.86
\end{array}\right] \\
& \lambda_{\mathrm{UEP}}=\left[\begin{array}{r}
5.63 \\
-9.00 \\
0 \\
-1.47 \\
-2.58+j 8.71 \\
-2.58-j 8.71
\end{array}\right]
\end{aligned}
$$

\section{CONCLUDING REMARKS}

An approach to small-signal analysis and modal identification has been proposed. Various aspects have been illustrated on a simple 3-machine system, and a priori interesting features have been observed.

Such an interesting result concerns computation of system's unstable equilibrium points (UEPs), generally considered to be a problematic issue. These UEPs are found to be close to the corresponding SIME's UEPs, which are extremely easy to compute. Conceptually, this result is quite sound, since by essence SIME reproduces faithfully the dynamic behavior of the multimachine system. From a practical viewpoint, this result may greatly facilitate the search of system's UEPs, since the efficiency of a minmization procedure depends on the good choice of starting points.
Note, however, that such a minimization procedure becomes even unnecessary if the purpose is to compute system's eigenproperties, given the following observations.

The eigenvectors computed from SIME's UEPs are found to be very close to those computed from the system's UEPs.

Similarly, participation factors (PFs) computed from system's UEPs and PFs computed from SIME's UEPs are very close to each other. Note, however, that PFs computed for identifying the machines' influence on transient stability may advantageously be replaced by SIME's assessment of critical machines and their degree of criticality, which is computationally significantly more straightforward.

Concerning explorations of damping influence, it is suggested to use the corresponding OMIB swing curve which provides a considerably clearer description of the phenomena and a much easier means of analysis.

More generally, SIME shows to be quite helpful for modal identification purposes: it determines unambiguously the modes of oscillation as well as the involved machines and their respective influcence. Note that all aspects relating to the dynamic, non linear system's behavior have long been validated through extensive simulations.

Admittedly, the above linearized analysis aspects would deserve further validation, performed on real world power systems with detailed modeling.

\section{REFERENCES}

[1] Task Force 07 of Advisory Group 01 of Study Committee 38 (1996): "Analysis and control of power system oscillations". CIGRE final report.

[2] V. Vittal, N. Bhatia, and A.A. Fouad, "Analysis of the inter-area mode phenomenon in power systems following large disturbances", IEEE Trans. on PWRS, Vol. 6, No. 4, November 1991.

[3] B. Berggren, and G. Andersson, "On the nature of unstable equilibrium points in power system", IEEE Trans. on PWRS, Vol. 8, No. 2, 1993, pp. 738-745.

[4] J.L. Jardim, B. Cory, and N. Martins, "Efficient transietn stability assessment using transient energy function". Proceedings of the 13th PSCC, Trondheim, Norway, June 28-July 2nd, 1999.

[5] A. Roth, "Transient stability assessment and control by modal analysis", University of Liège and Royal Institute of Technology, Dec. 2000 (65 pages).

[6] Y. Zhang, L. Wehenkel, P. Rousseaux, and M. Pavella, M., "SIME: A hybrid approach to fast transient stability assessment and contingency selection", Int. Journal of Electrical Power and Energy Systems, Vol. 19, No. 3, 1997, pp. 195-208.

[7] M. Pavella, D. Ernst, and D. Ruiz-Vega, Transient stability of power systems: a unified approach to assessment and control, Kluwer Academic Publishers, September 2000.

[8]G. Rogers. "Power System Oscillations” Kluwer Academic Publishers, 2000.

[9] P. Kundur. "Power System Stability and Control. McGraw Hill, 1994

[10] EPRI (Electric Power Research Institute), 1991: "Evaluation of transient energy method software for dynamic security analysis". Final EPRI Report No. EPRI EL-7357, Project 4000-18, July 1991.

[11] M.J. Gibbard, N. Martins, J. Sanchez-Gasca, N. Uchida, V. Vittal, and L. Wang, "Recent applications of linear analysis techniques", IEEE Trans. on Power systems, Vol. 16, No. 1, February 2001.

[12] J. Chow. "Power System Toolbox Vesion 2.0: A set of coordinated m-files for use with MATLAB”. Cherry Tree Scientific Software. Ontario, 1997. 